\title{
H. Sensor proteins
}

\section{H-01 \\ Metal sensing transcriptional repressors from bacterial pathogens \\ David P. Giedroc}

Department of Chemistry, Indiana University, Bloomington, IN 47405-7102, USA. giedroc@indiana.edu

Metal sensor proteins control the expression of genes that allow organisms to quickly adapt to chronic toxicity or deprivation of both biologically essential metal ions and heavy metal pollutants found in their microenvironment. Accumulating evidence suggests that metal ion homeostasis and resistance define an important tug-of-war in human host-bacterial pathogen interactions. This adaptive response originates with the formation of metal coordination complexes of exquisite selectivity that drives conformational switching within an oligomer. In this presentation, recent efforts to understand the structural and physical basis of how metal binding drives allosteric regulation of operator DNA binding by a number of metal sensor proteins will be discussed. These include a paradigm ArsR/SmtB family $\mathrm{Zn} / \mathrm{Co}$ efflux regulator from $S$. aureus, CzrA, a Cu-specific sensor from Mycobacterium tuberculosis, CsoR, and a Zn-specific metal uptake regulator from Streptococcus pneumoniae, AdcR. General features of the rules that appear to govern functional (vs. non-functional) allosteric switching of metal sensor proteins will be discussed. Supported by the US NIH (GM042569).

\section{H-02}

\section{Metal sensors of Synechocystis PCC 6803: what defines} selectivity?

Nigel J. Robinson, Kevin J. Waldron, Carl J. Patterson, Samantha J. Dainty

Cell and Molecular Biosciences, University of Newcastle, Newcastle upon Tyne NE2 4HH, UK. n.j.robinson@ncl.ac.uk

The cyanobacterium Synechocystis PCC 6803, in common with most bacteria, contains multiple DNA binding, metal binding, metal sensors. These include the MerR-like protein CoaR and the SmtB-like protein ZiaR. CoaR is an activator which under-winds the sub-optimally spaced operator-promoter region of a gene encoding a cobaltexporter CoaT in response to metal binding, while ZiaR dissociates to de-repress the operator promoter of a gene encoding the zinc-exporter $\mathrm{ZiaA}$ in response to metal binding. Mutants deficient in the two coa genes are sensitive to cobalt but not to zinc while mutants missing the two zia genes are sensitive to zinc but not to cobalt. Quadruple mutants are no more sensitive to zinc or cobalt than the respective double mutants. Chimeric constructs in which the cobalt exporter is placed under the control of $\mathrm{ZiaR}$ give negligible resistance to cobalt. In contrast, when the zinc exporter is placed under the control of CoaR, a substantial proportion of the zinc resistance seen in wild-type cells is re-introduced. This implies that, at least at the level of metal selection acting on living cells, ZiaR is specific for zinc and not for cobalt while CoaR is relatively non-specific and capable of conferring resistance in response to either metal. This conclusion is supported by assays of gene expression mediated by each regulator in response to cobalt and zinc obtained using RT-PCR and reporter gene constructs. In vitro metal-binding and DNA-binding studies of recombinant ZiaR will be reported along with analyses of gene regulation mediated by CoaR in E. coli. In exact opposition to the previous conclusions, these data show that ZiaR is relatively non-specific and capable of responding to both metals while CoaR appears highly specific responding just to cobalt. Explanations for these seemingly conflicting observations will be provided.

\section{H-03}

\section{Coordinating intracellular nickel: the NikR and $R c n R$ repressor proteins}

Peter Chivers ${ }^{1}$, Jeffrey Iwig ${ }^{1}$, Michael Bradley ${ }^{1}$, Michael Maroney ${ }^{2}$

${ }^{1}$ Department of Biochemistry and Molecular Biophysics, Washington University, St. Louis, USA.

${ }^{2}$ Department of Chemistry, University of Massachusetts, Amherst, USA

Bacteria and archaea use nickel for various enzymatic activities. Nickel must be acquired from the growth environment by specific transporter proteins. If intracellular nickel levels exceed the binding capacity of the cell, then excess nickel is either stored or excreted from the cell. In E. coli, these processes are transcriptionally regulated by the NikR and RcnR repressors, which are both widely conserved proteins. NikR represses the expression of the Nik$\mathrm{ABCDE}$ nickel transporter, which imports nickel ions in complex with L-histidine. In some microbes, NikR regulates other genes in addition to those encoding nickel transporters. RcnR, a founding member of a new structural class of bacterial transcriptional regulator, represses expression of the RcnA nickel efflux protein. The NikR and RcnR repressors provide an excellent system with which to understand explore how the biophysical and biochemical properties of metalloregulators are coupled to distinct biological roles.

NikR and RcnR are both tetrameric but structurally unrelated. An important feature of both proteins is the ability to link Ni-binding site occupancy with DNA-binding affinity. NikR binds nickel(II) (4/tetramer) with very high affinity $\left(K_{\mathrm{d}} \sim 1 \mathrm{pM}\right)$ using square planar coordination geometry, which leads to increased DNA-binding affinity. RcnR binds nickel(II) and cobalt(II) with lower affinity $\left(K_{\mathrm{d}} 5-25 \mathrm{nM}\right)$ using a six-coordinate binding site that uses two main chain N-ligands. Metal binding to RcnR decreases DNA-binding affinity. Both NikR and RcnR use a conserved Cys-residue within the metal-binding site to coordinate nickel(II), but the residue plays different roles in the two proteins. The differences in the nickelbinding properties of NikR and RenR are consistent with their opposing functions in response to changes in intracellular nickel ion levels. 


\section{H-04}

Iron-containing sensors and the response to NO in Escherichia coli

\section{Stephen Spiro}

Department of Molecular and Cell Biology, The University of Texas at Dallas, 800 W Campbell Road, Richardson, TX 75080, USA.

stephen.spiro@utdallas.edu

E. coli can be exposed to the nitric oxide (NO) synthesized endogenously by the one-electron reduction of nitrite, or (in the case of some pathogenic strains) to the NO made by the host iNOS. NO is growth inhibitory most likely due to the inhibition of enzymes containing $[\mathrm{Fe}-\mathrm{S}]$ clusters. E. coli expresses three enzymes that detoxify NO: flavohemoglobin (Hmp) oxidizes NO to nitrate; flavorubredoxin (FIRd) reduces $\mathrm{NO}$ to $\mathrm{N}_{2} \mathrm{O}$; and the respiratory nitrite reductase (Nrf) is also an $\mathrm{NO}$ reductase that reduces $\mathrm{NO}$ to $\mathrm{NH}_{3}$. Expression of the genes encoding these inducible defenses is coordinated by two NO-sensing transcriptional regulators, NorR and NsrR.

NorR is an activator of the genes encoding FlRd, and contains a mono-nuclear non-heme iron that is the binding site for NO. Formation of a mono-nitrosyl iron species stimulates NorR to make a productive interaction with RNA polymerase that results in transcription activation. Recent studies employing mutagenesis, spectroscopy and structural modeling have identified the likely iron ligands in the N-terminal GAF domain of NorR. NsrR is a transcriptional repressor of the gene encoding Hmp, and of the operon that encodes Nrf. Recent studies from other labs have shown that the NsrR homologues from Bacillus subtilis, Streptomyces coelicolor and Neisseria gonorrhoeae contain $[\mathrm{Fe}-\mathrm{S}]$ clusters, which may be either $[2 \mathrm{Fe}-2 \mathrm{~S}]$ or $[4 \mathrm{Fe}-4 \mathrm{~S}]$; presumably NsrR is inhibited by nitrosylation of the cluster. We have used high-throughput techniques to define the NsrR regulon of $E$. coli. These analyses have shown that NsrR regulates a diverse array of activities including $[\mathrm{Fe}-\mathrm{S}]$ cluster repair and biogenesis, motility, aromatic amine degradation and carbon and energy metabolism. In this presentation, I will provide an update of our current understanding of the mechanisms of NorR and NsrR, as well as the physiological roles of the genes under their control.

\section{H-05}

\section{A "turn-on" FRET-based biosensor for NO detection} Maria Strianese $^{1}$, Francesco De Martino', Vincenzo Pavone ${ }^{2}$, Angela Lombardi ${ }^{2}$, Gerard W. Canters ${ }^{3}$, Claudio Pellecchia ${ }^{1}$ ${ }^{1}$ Department of Chemistry, Salerno University, 84084 Fisciano, Italy. ${ }^{2}$ Department of Chemistry, Naples University, 80126 Naples, Italy. ${ }^{3}$ Leiden Institute of Chemistry, Leiden University, 2300 RA Leiden, The Netherlands.mstriane@unisa.it

NO plays a crucial role in a variety of biological processes. It regulates vasodilatation in the circulatory system and long-term potentiation in the brain. On the other hand, micromolar concentrations of NO can lead to carcinogenic and neurodegenerative disorders. Therefore, there is a pressing need for methods to detect NO [1]. The techniques commonly used for NO detection, due to the limitations of low sensitivity or expensive instrumentation, are not generally useful, especially in biological settings [1]. Recently, we and others proposed a novel use of FRET to monitor the activity of a donor-acceptor pair on a protein, opening the doors to a new generation of fluorescence-based biosensors [2]. Herein, we extended the FRET-based approach to cytochrome $c$ peroxidase $(\mathrm{CcP})$ for developing a "turn-on" [1] FRET-based NO biosensor.

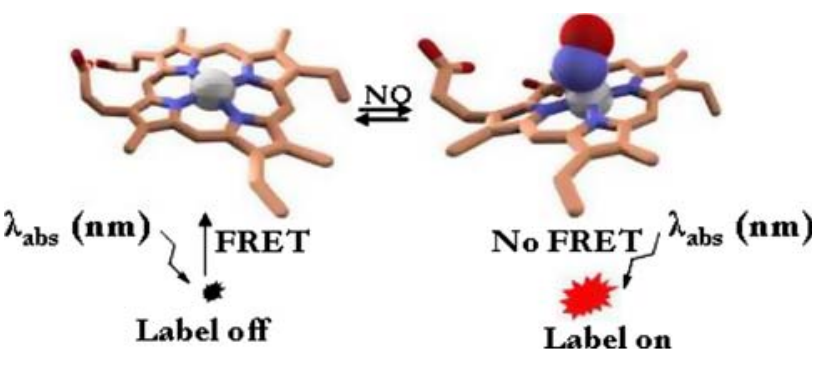

References

1. Lim MH, Lippard SJ (2007) Acc Chem Res 40:41

2. Zauner G, Strianese M, Bubacco L, Aartsma TJ, Tepper AWJW, Canters GW (2008) Inorg Chim Acta 361:1116

\section{H-06}

$\mathrm{Mg}^{2+}$-dependent gating of MgtE channel underlying

$\mathrm{Mg}^{2+}$ homeostasis

Motoyuki Hattori $^{1}$, Ryuichiro Ishitani ${ }^{1}$, Osamu Nureki ${ }^{1}$

${ }^{1}$ Department of Basic Medical Science, Institute of Medical Science, The University of Tokyo, 4-6-1 Shirokanedai, Tokyo 108-8639,

Japan.nureki@ims.u-tokyo.ac.jp

Magnesium ion $\left(\mathrm{Mg}^{2+}\right)$ is an essential metal element for life that plays a crucial role in many biological processes. However, among the major biological cations, the mechanisms of $\mathrm{Mg}^{2+}$ transport and homeostasis are only slowly being elucidated. The MgtE family of $\mathrm{Mg}^{2+}$ transporters is ubiquitously distributed in all the three kingdoms of life. Recently, we solved the crystal structures of the full-length Thermus thermophilus MgtE at 3.5 A resolution and the cytosolic domain in the presence and absence of $\mathrm{Mg}^{2+}$ at 2.3 and $3.9 \AA$ resolutions, respectively, which showed that the drastic $\mathrm{Mg}^{2+}$-dependent structural transition involving the plug (connecting) helices. Based on these structures, we proposed a $\mathrm{Mg}^{2+}$ homeostasis mechanism, in which the cytosolic domain of MgtE acts as a ' $\mathrm{Mg}^{2+}$ sensor', which regulates the gating of the ion-conducting pore by sensing the intracellular $\mathrm{Mg}^{2+}$ concentration. However, no complementary functional analyses to support the proposed model have yet been reported. Here, we describe the channel properties and the $\mathrm{Mg}^{2+}$-dependent cooperative gating mechanism of MgtE, by a combination of X-ray crystallographic, genetic, biochemical and electrophysiological analyses, which directly underlie the mechanism of $\mathrm{Mg}^{2+}$ homeostasis by MgtE.

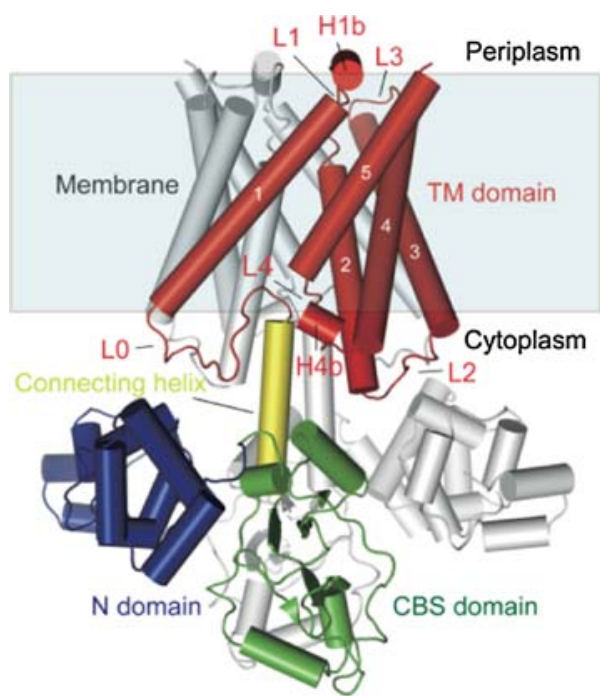




\section{H-07}

\section{Ni-specific sensing by a Fur homologue Nur Jung-Hye Roe}

School of Biological Sciences, Institute of Microbiology,

Seoul National University, Seoul 151-742, Korea.jhroe@snu.ac.kr Members of ferric-uptake-regulator (Fur) family proteins are the most wide-spread metal sensors and regulators for metal homeostasis in bacteria. They show a remarkable versatility in sensing metals as well as some oxidants. How they specifically bind metals and regulate its DNA binding activity is beginning to get revealed. Nickel serves as a cofactor for various microbial enzymes such as urease, hydrogenase, glyoxalase, and superoxide dismutase (SOD). From an effort to find a nickel-responsive regulator that represses Fe-containing SOD but induces Ni-containing SOD in Streptomyces coelicolor, we identified a Fur homologue, Nur, which binds directly to the promoter region of the $\operatorname{sod} F$ gene encoding FeSOD in the presence of nickel. Nur also regulates at least two putative nickel transporter operons, contributing to nickel homeostasis. The crystal structure of Nur reveals a unique nickel-specific metal site in addition to a non-specific common metal site. We identified the 6-5-6 motif of the Nur recognition box, which matches very well with a Nur/DNA complex model. The interaction mode shows a remarkable difference from a model for Fur with $n-1-n$ type of recognition sequence. The observation that the specificity of Nur in metal sensing and DNA recognition is conferred by the specific metal site suggests that its introduction drives the evolution of Nur orthologs in the Fur family.

\section{References}

1. Ahn BE, Cha J, Lee EJ, Han AR, Thompson CJ, Roe JH (2006) Mol Microbiol 59:1848-1858

2. An YJ, Ahn BE et al (2009) Nucleic Acids Res (in press)

\section{H-08}

\section{Single-molecule dynamics of metal regulators and transporters \\ Peng Chen}

Department of Chemistry and Chemical Biology, Cornell University, Ithaca, NY 14853, USA. pc252@cornell.edu

To maintain normal metal metabolism, organisms utilize dynamic cooperation of many biomacromolecules for regulating metal ion concentrations and bioavailability; how these biomacromolecules work together to achieve their functions is largely unknown, however. For example, how do metalloregulators and DNA interact to control gene expression to maintain healthy cellular metal level? How do metal transporters collaborate to deliver metal ions?

We have been studying the macromolecular machineries for metal regulation and transport at the singe-molecule level. Some highlights of our recent work include: (1) The invention of engineered DNA Holliday junctions as general single-molecule reporters for proteinDNA interactions, especially those involving only subtle DNA structural changes. We showed how a $\mathrm{Pb}^{2+}$-responsive regulator binds to DNA, changes DNA structure, constrains DNA conformational flexibility, and alters DNA dynamics for transcriptional regulation. (2) The development of nanovesicle trapping coupled with single-molecule fluorescence resonance energy transfer (smFRET) as a powerful and general tool for studying weak, transient protein interactions. We showed how the copper chaperone Hah1 and a single domain of the Wilson disease protein (WDP) interact dynamically, capturing distinct intermediates and resolving intermediate interconversion dynamics that facilitate their copper transfer.

\section{References}

1. Sarkar SK, Andoy NM, Benítez JJ, Chen PR, Kong JS, He C, Chen P (2007) J Am Chem Soc 129:12461-12467
2. Benítez JJ, Keller AM, Ochieng P, Yatsunyk LA, Huffman DL, Rosenzweig AC, Chen P (2008) J Am Chem Soc 130:2446-2447

3. Chen P, Andoy NM (2008) Inorg Chim Acta 361:809-819

\section{H-09}

Structure of the [2Fe-2S] transcriptional activator

SoxR

Satoshi Watanabe ${ }^{1}$, Akiko Kita ${ }^{1}$, Kazuo Kobayashi ${ }^{2}$, Kunio Miki ${ }^{1,3}$

${ }^{1}$ Department of Chemistry, Graduate School of Science, Kyoto University, Kyoto 606-8502, Japan.

${ }^{2}$ ISIR, Osaka University, Osaka 567-0047, Japan.

${ }^{3}$ RIKEN SPring-8 Center at Harima Institute, Hyogo 679-5148, Japan.watanabe@kuchem.kyoto-u.ac.jp

SoxR functions as a sensor of oxidative stress. It contains a [2Fe-2S] cluster and the redox states of the $[2 \mathrm{Fe}-2 \mathrm{~S}]$ cluster regulate the activity of SoxR. SoxR belongs to the MerR family of transcriptional activators, target promoters of which have an unusual long spacer between the -35 and -10 operator elements. In the active state, SoxR and other MerR family proteins activate transcription from unique promoters by distorting the DNA conformation. In order to elucidate the redox-dependent transcriptional activation mechanism of SoxR, we have determined the crystal structures of SoxR and its complex with DNA in the oxidized (active) state [1].

The overall structure of SoxR consists of a DNA-binding domain, a dimerization helix and an Fe-S cluster-binding domain. The dimerization helix forms an antiparallel coiled coil, stabilizing the SoxR dimer. The structures reveal that the $[2 \mathrm{Fe}-2 \mathrm{~S}]$ cluster of SoxR is unusually solvent exposed and surrounded by an asymmetric environment, suggesting that the asymmetrically charged environment is a key factor of redox-dependent conformational changes of SoxR. The DNA structure is shown to be sharply bent at the middle and unwound by 3-bp, compared to a B-form DNA. Based on comparison of the target promoter sequences of the MerR family, the present structures show an activated promoter conformation with a 20 -bp spacer in the MerR family.

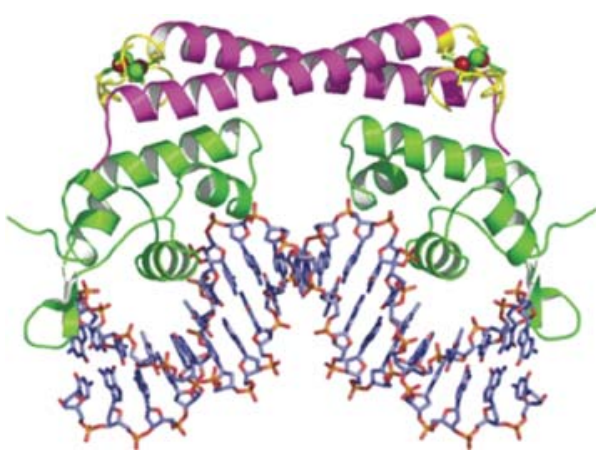

Reference

1. Watanabe S, Kita A, Kobayashi K, Miki K (2008) Proc Natl Acad Sci USA 105:4121-4126

\section{H-10}

Tunable oxygen sensing by the $E$. coli transcriptional regulator FNR

Andrew J. Thomson', Jason C. Crack ${ }^{1}$, Nick Le Brun', Adrian J. Jervis ${ }^{2}$, Jeff Green ${ }^{2}$ 
${ }^{1}$ Centre for Molecular and Structural Biochemistry (CMSB), School of Chemical Sciences and Pharmacy, University of East Anglia, Norwich NR4 6XZ, UK.

${ }^{2}$ Department of Molecular Biology and Biotechnology, Firth Court, University of Sheffield, Sheffield S10 2TN, UK.

a.thomson@uea.ac.uk

FNR, the fumarate nitrate reduction regulator, is a global transcription factor controlling gene expression in response to $\mathrm{O}_{2}$ levels. Sensing entails a $[4 \mathrm{Fe}-4 \mathrm{~S}]^{2+}$ cluster reacting with $\mathrm{O}_{2}$ to generate a $[2 \mathrm{Fe}-2 \mathrm{~S}]^{2+}$ form via an intermediate $[3 \mathrm{Fe}-4 \mathrm{~S}]^{1+}$ cluster. The initial product of $\mathrm{O}_{2}$ reduction, the superoxide ion, is rapidly recycled back in vivo to $\mathrm{O}_{2}$ and $\mathrm{H}_{2} \mathrm{O}$ by SOD, peroxidase and FNR itself. This may provide a mechanism to amplify the sensitivity of FNR towards $\mathrm{O}_{2}$. The mutation $\mathrm{S} 24 \mathrm{~F}$, adjacent to a cluster ligand $\mathrm{C} 23$, strongly enhances the stability of the $[4 \mathrm{Fe}-4 \mathrm{~S}]^{2+}$ cluster in the presence of $\mathrm{O}_{2}$ showing how this sensor protein in vivo can adjust its $\mathrm{O}_{2}$ sensitivity depending on the oxygen levels in the micro-environment in which the organism lives.

\section{References}

1. Crack JC et al (2004) J Biol Chem 279:9278

2. Crack JC et al (2006) J Biol Chem 281:18909

3. Crack JC et al (2007) PNAS USA 104:2092

4. Crack JC et al (2008) JACS 130:1749

5. Jervis AJ et al (2009) PNAS USA (in press)

\section{H-11}

\section{Insights into signal transduction mechanism of $\mathrm{O}_{2}$} sensor protein, $E c D O S$, by resonance Raman spectroscopy

Samir F. El-Mashtoly ${ }^{1}$, Toru Shimizu², Teizo Kitagawa ${ }^{3}$

${ }^{1}$ King Khalid University, Abha, Saudi Arabia.

${ }^{2}$ Tohoku University, Sendai, Japan.

${ }^{3}$ Toyota Physical and Chemical Research Institute, Nagakute, Aichi 480-1192, Japan. samirfathi@ hotmail.com

The direct oxygen sensor protein from E. coli (EcDOS) is a hemebased signal transducer protein responsible for phosphodiesterase (PDE) activity. Binding of $\mathrm{O}_{2}, \mathrm{CO}$, or $\mathrm{NO}$ to heme significantly enhances the PDE activity toward c-di-GMP. We have investigated the resonance Raman (RR) spectra for wild-type (WT) and several mutants of heme-containing PAS domain of EcDOS. The UVRR spectra indicate that binding of $\mathrm{O}_{2}$ or $\mathrm{CO}$ to WT exhibited drastic changes in a situation of Tyr126. It is deduced from these results that Asn84 forms a hydrogen bond with Tyr126 in either the $\mathrm{O}_{2}$ or CO bound forms but not in the ligand-free form. The PDE activities of ligand bound forms for N84V and Y126F mutants are significantly reduced, suggesting the significance of the hydrogen-bonding network from heme 6-propionate to Tyr126 through Asn84 in signaling process.

The RR spectra revealed the presence of direct interactions of Arg97 and Phe113 with a heme-bound ligand. Profound structural changes in the heme propionates were detected upon dissociation of $\mathrm{CO}$, and the mutation of Arg97 and Phe113 significantly influenced the transient binding of Met 95 to the heme upon photodissociation of CO [2]. This implies that the electrostatic interaction of Arg97 and steric interaction of Phe113 are crucial for regulating the competitive recombination of Met95 and $\mathrm{CO}$ to the heme. Based on these results, we propose a model for the role of the heme propionates in communication between the heme and the protein moiety.

\section{References}

1. El-Mashtoly SF, Takahashi H, Shimizu T, Kitagawa T (2007) J Am Chem Soc 129:3556-3563

2. El-Mashtoly SF, Nakashima S, Tanaka A, Shimizu T, Kitagawa T (2008) J Biol Chem 283:19000-19010
H-12

Iron response regulator protein is a self-oxidative oxidase-transcription factor: a mechanism for heme-mediated self-oxidation

Koichiro Ishimori

Department of Chemistry, Faculty of Science, Hokkaido University, Sapporo 060-0810, Japan. koichiro@sci.hokudai.ac.jp

The Bradyrhizobium japonicum transcriptional regulator Irr (iron response regulator) degrades in response to heme binding via a mechanism that involves oxidative modification of the protein. Here, we show that heme-bound Irr catalyzes $\mathrm{H}_{2} \mathrm{O}_{2}$ formation from molecular oxygen as found for myoglobin. Interestingly, the peptide of Irr was oxidized by $\mathrm{H}_{2} \mathrm{O}_{2}$, while that of myoglobin was not oxidized at all, indicating that the generated $\mathrm{H}_{2} \mathrm{O}_{2}$ was further converted into a more reactive form. Such activation of $\mathrm{H}_{2} \mathrm{O}_{2}$ was also encountered for the bacterial $\mathrm{H}_{2} \mathrm{O}_{2}$ sensor protein, PerR, containing non-heme iron, and the inductively coupled plasma atomic emission spectrometry and mass spectrometry clearly demonstrate that Irr also contains non-heme iron. In oxidized Irr, ${ }^{63} \mathrm{His}$, corresponding to one of the histidine coordinated to the non-heme iron in PerR, was identified as the oxidation site. From these results, we can conclude that $\mathrm{H}_{2} \mathrm{O}_{2}$ is subsequently converted to a more reactive form by nonheme iron in Irr, leading to the oxidation of the ligand to the nonheme iron, ${ }^{63} \mathrm{His}$. Thus, we, for the first time, demonstrate that Irr is a novel transcription factor showing heme and non-heme iron-dependent self-oxidative oxidase activity, where molecular oxygen is converted into a reactive oxygen species with the two-step activation to lead to the oxidation of own pept.

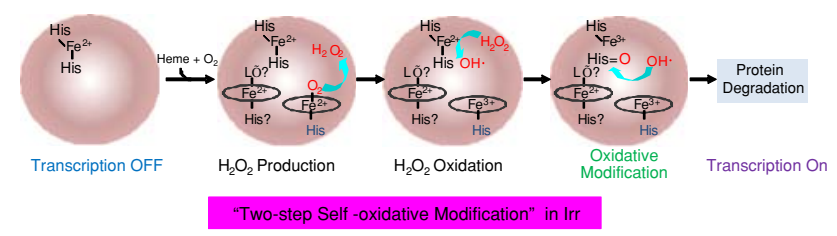

\section{H-13}

Heme-regulated eIF2 $\alpha$ kinase in the regulation of stress erythropoiesis

Jane-Jane Chen

Harvard-MIT Division of Health Sciences and Technology,

Massachusetts Institute of Technology, Cambridge, MA, USA.

j-jchen@mit.edu

Maturation of erythroid precursors requires active synthesis of a very large amount $(350 \mathrm{mg} / \mathrm{ml})$ of hemoglobin, which consists of two pairs of $\alpha$ - and $\beta$-globin subunits with each monomer bound to a heme moiety. Excess of any of these three components of hemoglobin is cytotoxic. Heme-regulated eIF2 $\alpha$ kinase (HRI) balances synthesis of heme and globin by sensing the intracellular heme concentration through its two heme-binding domains. Dissociation of heme from HRI in heme deficiency leads to its activation by autophosphorylation. Activated HRI phosphorylates the $\alpha$-subunit of eukaryotic initiation factor (eIF $2 \alpha$ ), which impairs the recycling of eIF2 for translational initiation and results in cessation of protein synthesis. In the absence of HRI as in $\mathrm{Hri}^{--}$mice, uncontrolled protein synthesis in heme deficiency results in globin aggregation and precipitation in the red cell and its precursors. Beyond heme deficiency, HRI is also the predominate eIF $2 \alpha$ kinase activated by arsenite-induced oxidative stress, heat shock and UV irradiation in erythroid cells. Thus, HRI may protect erythroid cells against stress generally and may play a role in the physiological response to intrinsic disorders of red blood cells. Indeed, HRI deficiency in mice exacerbates erythropoietic protoporphyria and renders $\beta$-thalassemia embryonically lethal. In addition to general 
inhibition of protein synthesis, phosphorylation of eIF $2 \alpha$ by HRI also leads to a selective increased translation of ATF4 mRNA in erythroid precursors and the subsequent induction of gene transcription for the adaptation to chronic iron deficiency. $\mathrm{Hri}^{-1-}$ mice develop ineffective erythropoiesis in iron/heme deficiency with an inhibition of erythroid differentiation at the basophilic stage, consistent with the increased HRI expression during erythroid differentiation at this stage.

\section{H-14}

Heme-sensing mechanisms of heme-sensor proteins associated with protein synthesis and circadian rhythms Jotaro Igarashi, Motohiko Murase, Marketa Martinkova, Kenichi Kitanishi, Toru Shimizu

Institute of Multidisciplinary Research for Advanced Materials, Tohoku University, Sendai 980-8577, Japan.

shimizu@tagen.tohoku.ac.jp

Heme per se may be the first signal, and thus plays a regulatory role, in various important physiological functions. Heme-regulated eukaryotic initiation factor $2 \alpha$ (eIF2 $\alpha$ ) kinase (HRI) plays a role in response to $\mathrm{Fe}(\mathrm{III})$-protoporphyrin IX complex (hemin) deficiency. The molecular mechanism of heme sensing by HRI, including identification of the specific heme binding site, has been elucidated [1-3]. It was suggested that heme regulation of HRI was induced by interactions between heme and the catalytic domain, in conjunction with global tertiary structural changes in the $\mathrm{N}$-terminal domain accompanying heme coordination, thus not merely by coordination of heme iron with amino acids on a protein surface patch. Interestingly, cysteine thiolate is the heme-binding site of the heme-regulated transcription factors NAPS2 and mPer2 (both associated with circadian rhythms) [4-6]. Fast heme dissociation rates and redoxdependent ligand switches of heme iron appear to be shared by these proteins. Other heme-sensor proteins such as Bach1, IRP2, E75, DGCR8, and Slo BK channel also have cysteine thiolate as hemesensing sites. Therefore, we suggest that heme sensing is achieved by exploiting specific characteristics of cysteine thiolate, such as weak binding to $\mathrm{Fe}$ (III) heme and even weaker binding to $\mathrm{Fe}$ (II) heme. We further discuss global protein structural changes necessary for heme sensing by these heme-sensor proteins.

\section{References}

1. Igarashi J et al (2004) J Biol Chem 279:15752

2. Miksanova M et al (2006) Biochemistry 45:9894

3. Igarashi J et al (2008) J Biol Chem 283:18782

4. Uchida T et al (2005) J Biol Chem 280:21358

5. Mukaiyama Y et al (2006) FEBS J 273:1210

6. Kitanishi K et al (2008) Biochemistry 47:6157

\section{H-15}

Early events in heme-based sensor proteins studied by ultrafast optical spectroscopy Ursula Liebl ${ }^{1,2}$, Taku Yamashita ${ }^{1,2}$, Latifa Bouzhir-Sima ${ }^{1,2}$, Jean-Christophe Lambry ${ }^{1,2}$, Marten H. Vos ${ }^{1,2}$

${ }^{1}$ Laboratoire d'Optique et Biosciences, CNRS, Ecole Polytechnique, 91128 Palaiseau, France.

${ }^{2}$ INSERM U696, 91128 Palaiseau, France. marten.vos@polytechnique.edu

Heme-based sensor proteins detect changes in the level of diatomic gaseous ligands. In these proteins, binding of the messenger molecules (essentially $\mathrm{NO}, \mathrm{CO}$ or $\mathrm{O}_{2}$ ) to heme initiates chemistry that eventually results in the organism's response to changes in ligand availability. Within the protein, ligand binding to, or dissociation from, the heme leads to modulation or switching of the activity of an associated enzymatic domain. Thus, dissociation of the ligand using light pulses in principle can trigger the signaling process and may permit characterization of short-lived intermediates in the intra-protein signaling pathway. Using femtosecond light pulses, processes on the intrinsic timescale of intra-protein conformational motions can be studied that are also accessible by molecular dynamics simulations [1]. An overview of our recent studies on native and engineered heme domains of the oxygen sensors FixL from B. japonicum and Dos from $E$. coli will be given with emphases on (a) effective caging of oxygen in the heme pocket specifically in these proteins, and (b) the possibility to photodissociate not only external but also internal ligands from six-coordinate proteins, which allows activation of processes involved in switching towards the external-ligand bound state. In the latter way, we have shown that the flexibility of the methionine side chain coordinating the heme group in $E c$ Dos plays an important role in initial events leading to a switch in the heme domain that allows $\mathrm{O}_{2}$ to bind [2].

\section{References}

1. Vos MH (2008) Biochim Biophys Acta 1777:15-31

2. Yamashita T, Bouzhir-Sima L, Lambry J-C, Liebl U, Vos MH (2008) J Biol Chem 283:2344-2352

\section{H-16 \\ Molecular oxygen regulates the enzymatic activity of a heme-containing diguanylate cyclase, HemDGC Shigetoshi Aono}

Okazaki Institute for Integrative Bioscience, National Institutes of Natural Sciences, 5-1 Higashiyama, Myodaiji, Okazaki 444-8787, Japan. aono@ims.ac.jp Bis- $\left(3^{\prime}, 5^{\prime}\right)$-cyclic di-guanosine monophosphate (c-di-GMP) is a bacterial second messenger responsible for cell motility, differentiation, development, virulence, and biofilm formation. Low concentrations of c-di-GMP promote motile growth of bacteria, while high concentrations promote sessile growth with biofilm formation. The balance of the formation and hydrolysis of c-di-GMP controls intracellular concentrations of c-di-GMP. The formation of c-di-GMP is catalyzed by diguanylate cyclase (DGC) that forms c-di-GMP from two molecules of GTP. DGC has a conserved sequence motif named GGDEF motif.

In this work, we have studied the biochemical and biophysical properties of a heme-containing diguanylate cyclase (HemDGC) from an obligatory anaerobic bacterium Desulfotalea psychrophila. HemDGC consists of a globin and GGDEF domains that act as a sensor and enzymatic domains, respectively. The globin domain of HemDGC contains a protoheme being a five-coordinate with the proximal His ligand in the resting state. The enzymatic activity of HemDGC is regulated by $\mathrm{O}_{2}$, i.e., only $\mathrm{O}_{2}$-bound $\mathrm{HemDGC}$ is active for the formation of c-di-GMP, but the ferrous, CO-bound, and ferric forms are not. Resonance Raman spectroscopy reveals that Tyr55 [position 10 in the $\mathrm{B}$ helix (B10)] is a key residue for the discrimination of $\mathrm{O}_{2}$ from $\mathrm{CO}$. Tyr55 forms a hydrogen bonding network with the hemebound $\mathrm{O}_{2}$, but not with $\mathrm{CO}$. The formation of the specific hydrogen bonding network at the distal heme pocket will cause a conformational change of HemDGC, which results in the activation of the DGC activity. $\mathrm{O}_{2}$ may act as an oxidative stress toward D. psychrophila, which induces the formation of c-di-GMP via HemDGC activation for the response to the oxidative stress. 